\title{
POLYMORPHISM OF THE CLINICAL SIGNS OF CELIAC DISEASE IN CHILDREN
}

\author{
Laura Mihaela Trandafir', Eugen Cirdeiu', Carmen Oltean', Doina Mihaila², \\ Dana-Teodora Anton-Paduraru ${ }^{1}$ \\ ${ }^{1} 3^{\text {rd }}$ Clinic of Pediatrics, "Gr. T. Popa" University of Medicine and Pharmacy, Iasi \\ ${ }^{2}$ Laboratory of Pathology, "Sf. Maria” Emergency Clinical Hospital for Childern, Iasi
}

\begin{abstract}
Celiac disease (CD) is an autoimmune systemic condition caused by sensitivity to gluten in genetically predisposed patients. The clinical signs of CD are extremely diverse, ranging from the typical intestinal malabsorption syndrome (characterized by chronic diarrhea, abdominal distension and malnutrition) to atypical symptoms that may involve any system or organ: chronic constipation, increased level of liver enzymes, iron deficiency anemia, recurrent abdominal pain, neurological conditions, tooth enamel erosion. The authors report four pediatric cases diagnosed with various atypical forms of celiac disease: the form associated with recurrent abdominal pain and increased level of liver enzymes, and the form associated with chronic constipation, all accompanied by failure to thrive and one case of type I diabetes mellitus associated with celiac disease. To conclude with, pediatricians, gastroenterologists and general practitioners should be familiar with all the clinical forms of coeliac disease in order to be able to diagnose this childhood disease and thus prevent long-term complications like osteoporosis, infertility and intestinal lymphoma.
\end{abstract}

Keywords: celiac disease, clinical signs, type I diabetes mellitus, child

\section{INTRODUCTION}

Celiac disease $(\mathrm{CD})$ is an autoimmune multisystemic condition triggered by gluten and related prolamins intake in genetically predisposed patients, which may impair any organ of system and which has a wide range of clinical signs of variable severity (1).

$\mathrm{CD}$ used to be considered a pediatric condition with specific gastrointestinal signs and symptoms due to intestinal malabsorption, consisting of chronic diarrhea, abdominal distension, anorexia, failure to thrive. The use of serologic screening tests (anti-gliadin, anti-endomisium, anti-tissue transglutaminase and anti-deamidated gliadin antibodies) allowed identifying all forms of CD: atypical, silent or latent, as well as disease identification among associated pathological conditions. Since it often has atypical symptoms (recurrent abdominal pain, constipation, iron deficiency anemia, increased level of liver enzymes, arthritis, etc.) or is clinically asymptomatic, CD may go undiagnosed or even entail complications if exposure to gluten continues. Knowledge of all forms of manifestation of the CD is vital for timely diagnosis setting before the occurrence of any complications that may impede upon the patients' life quality or even jeopardize their life altogether (intestinal lymphoma).

It is now known that $\mathrm{CD}$ onset may occur at any age, from infants to aged individuals, and have a polymorphic clinical presentation due mainly to its autoimmune component. Therefore, we report a series of clinical cases in whom $C D$ took the form of hepatic impairment, constipation, recurrent abdominal pain and one case in whom CD onset was associated with type 1 diabetes mellitus (type $1 \mathrm{DM}$ ) onset.

\section{Clinical case I}

5 years and 2 months old male patient hospitalized in the $3^{\text {rd }}$ Pediatric Department of the "Sf. Maria" Children Emergency Hospital of Iasi for lack

Corresponding author:

Laura Mihaela Trandafir, "Gr. T. Popa" University of Medicine and Pharmacy, 16 Universitatii Street, lasi

E-mail: trandafirlaura@yahoo.com 
of appetite, recurrent abdominal pain, weight gain decrement. The child had normal height and weight gain during the first two years of his life. On hospitalization he had a relatively good general state, $\mathrm{W}=13 \mathrm{~kg}, \mathrm{H}=119 \mathrm{~cm}$, BMI (body mass index) = 9.2, normal stethacoustic lung and heart functioning, lack of appetite, soft depressible abdomen, periumbilical spontaneous and palpation pain and physiological intestinal transit. Nevertheless, his behavioral disorders (short-tempered introverted child who did not fit in with the other children) attracted our attention. The biological tests performed revealed no inflammatory syndrome, hypochromic microcytic iron-deficiency anemia $(\mathrm{Hb}=10 \mathrm{~g} \%$, $\mathrm{VEM}=70 \mu 3, \mathrm{HEM}=28.5 \mathrm{pg}, \mathrm{CHEM}=36 \%$, serum $\mathrm{Fe}=25 \gamma \%$ ), and the titers of the anti-tissue transglutaminase antibodies (ATTG) IgA and IgG were pathological, as they were $53.5 \mathrm{UI} / 1$ and 13.4 UI/1, respectively. The presence of HLA DQ2 supported the diagnosis of $\mathrm{BC}$. The clinical-biological evolution was positive under a gluten-free diet. The patient regained his appetite, the abdominal pain disappeared, the weight gaining curve was slightly ascending and his behavioral disorders vanished. The ATTG antibodies were negative after 6 months of gluten-free diet (1.5 UI/1). After 8 months of gluten-free diet, the patient weighed $18 \mathrm{~kg}$, he was 121 $\mathrm{cm}$ tall and his $\mathrm{BMI}=12.3$.

\section{Clinical case II}

1 year and 6 months old female patient living in the countryside (in Botosani County) hospitalized in the $3^{\text {rd }}$ Pediatric Department of the "Sf. Maria" Children Emergency Hospital of Iasi for failure to thrive. According to her personal history, she is the second child of the family, delivered at term naturally (Weight on birth $=3,100$ grams), breastfed for 2 months and then fed with age-adapted Morinaga powder milk formula. Supplementary feeding was initiated at the age of 4 months and gluten-containing foods were added at the age of 5 months. Her personal medical history revealed her failure to thrive, which began at the age of 9 months. The patient's objective clinical examination revealed a relatively good general state, $\mathrm{Wb}=9 \mathrm{~kg},-2.15 \mathrm{SWD}$ (standard weight deviations) and $\mathrm{H}=83 \mathrm{~cm},-1.03$ SHD (standard height deviations), which were lower than the normal percentages specific to this age category. The paraclinical examinations conducted revealed no inflammatory syndrome, hypochromic microcytic iron-deficiency anemia $(\mathrm{HB}=9.3 \mathrm{~g} \%$, $\mathrm{VEM}=68 \mu 3, \mathrm{HEM}=23.3 \mathrm{pg}, \mathrm{CHEM}=34.6 \%$, serum $\mathrm{Fe}=30 \gamma \%$ ), increased level of liver enzymes with transaminase values three times higher than their normal value $(\mathrm{TGP}=83 \mathrm{UI} / 1, \mathrm{TGO}=92 \mathrm{UI} / 1)$. HBs antigen - negative, hepatitis $\mathrm{C}$ virus antibodies - negative, anti-cytomegalovirus antibodies negative were determined in order to describe the etiology of the increased level of liver enzymes. Anti-gliadin antibodies had pathological values $18 \mathrm{UI} / 1$ (VN below $6 \mathrm{UI} / 1)$. The duodenal intestinal biopsy (Fig. 1) revealed total villous atrophy, inflammatory lymphoplasmacytic infiltrate in the chorion, numerous intra-epithelial lymphocytes in the lamina (MARSH IIIC).

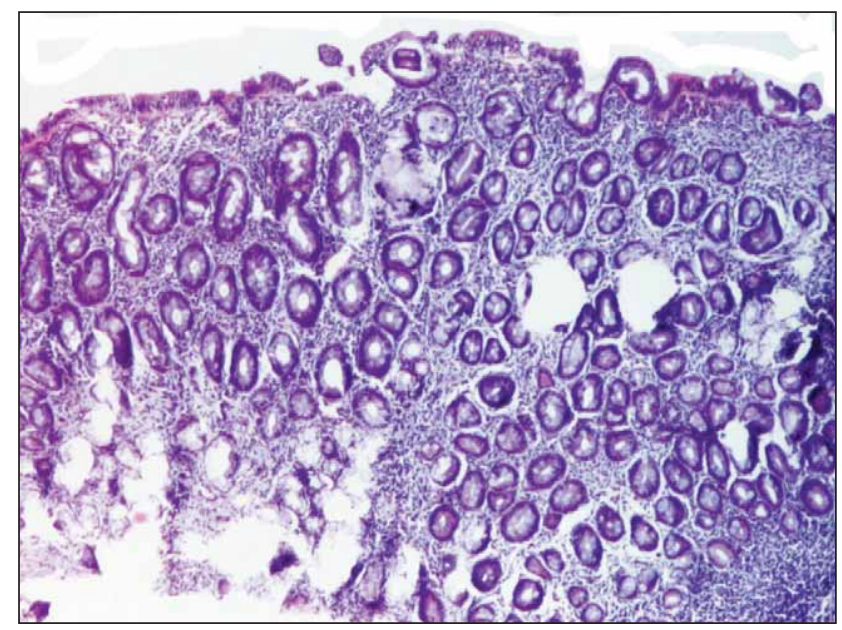

FIGURE 1. Total villous atrophy, inflammatory lymphoplasmacytic infiltrate in the chorion, numerous intra-epithelial lymphocytes in the lamina (MARSH IIIC)

The positive diagnosis was $\mathrm{CD}$ associated with reactive hepatopathy and iron deficiency anemia. After a gluten-free diet was initiated, the weight curve began to go up, and the TGP and TGO values became normal after 6 months of gluten-free diet.

\section{Clinical case III}

1 year and 2 months old male patient hospitalized in the $3^{\text {rd }}$ Pediatric Department of the "Sf. Maria" Children Emergency Hospital of Iasi on 31 August 2006 for failure to thrive and chronic constipation. His physical examination conducted on hospitalization revealed a relatively good general state, nutritional short stature ( $\mathrm{W}=7.5 \mathrm{~kg}, \mathrm{H}=76 \mathrm{~cm}$, PI $=0.68)$, pale teguments, reduced subcutaneous cell tissue on the abdomen, thorax and limbs, rickets sequelae (forehead humps, flared lower thorax, rachitic rosary), muscular hypotonia, lack of appetite, swollen abdomen, slow intestinal transit (highly consistent stools every 4-5 days). Laboratory tests revealed IgA anti-gliadin antibodies values of $38 \mathrm{UI} / 1$ (NV below $6 \mathrm{UI} / 1)$. Fist X-ray revealed delayed bone age as compared to the chronological 
age of 8 months. The endocrine examination ruled out congenital hypothyroidism (TSH, freeT4 - normal values, normal thyroid gland ultrasound scan). The pathology findings further to duodenal intestinal biopsy were (Fig. 2): subtotal villous atrophy, inflammatory lymphoplasmacytic infiltrate in the chorion, numerous intra-epithelial lymphocytes in the lamina - suggesting MARSH IIIB. In this case, the positive diagnosis was atypical $\mathrm{CD}$ associated with $2^{\text {nd }}$ degree protein-caloric malnutrition and constipation. After a gluten-free diet had been initiated, the weight curve began to go up, the intestinal transit was resumed and the IgA anti-gliadin antibodies values became normal after 3 months of gluten-free diet.

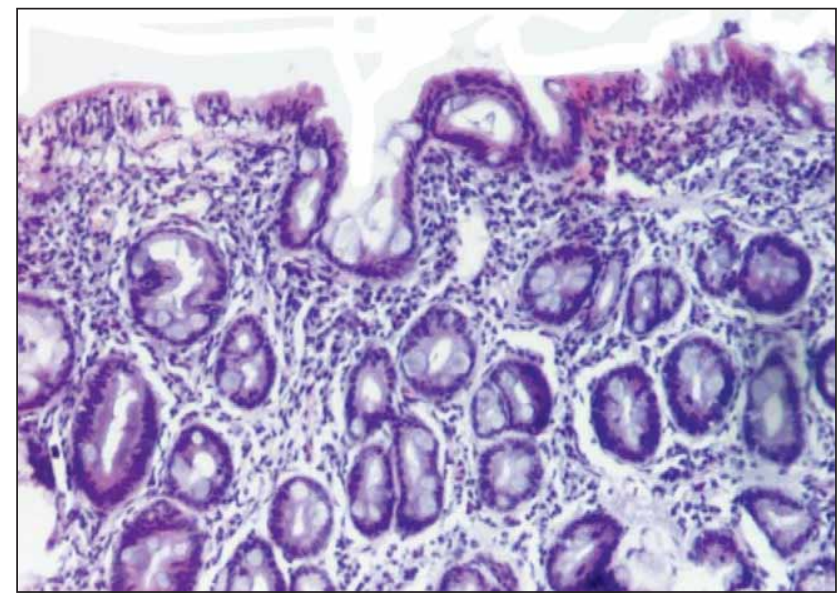

FIGURE 2. Subtotal villous atrophy, inflammatory lymphoplasmacytic infiltrate in the chorion, numerous intra-epithelial lymphocytes in the lamina (MARSH IIIB)

\section{Clinical case IV}

9 years and 7 months male patient hospitalized in the $3^{\text {rd }}$ Pediatric Department of the "Sf. Maria" Children Emergency Hospital of Iasi for altered general state and abdominal distension. On hospitalization, the child had altered general state, $\mathrm{W}=34 \mathrm{~kg}, \mathrm{H}=141 \mathrm{~cm}$, body mass index $(\mathrm{BMI})=$ 17.17 , swollen abdomen, the lower edge of the liver $3 \mathrm{~cm}$ below the costal margins, normal consistency liver (hepatomegaly), postprandial abdominal distension and occasional diffuse abdominal pain, stools full of mucus and undigested food, depigmented patches of skin on the upper and lower limbs. Paraclinical investigations revealed: no inflammatory syndrome, glycaemia $=207 \mathrm{mg} \%$ (NV $<120 \mathrm{mg} \%), \mathrm{HbA} 1 \mathrm{c}=13.53 \%(\mathrm{NV}<6 \%)$, glycosuria $=150 \mathrm{mg} / \mathrm{dl}$ and ketone bodies + , cholesterol $=$ $202 \mathrm{mg} / \mathrm{dl}$, triglycerides $=98 \mathrm{mg} \%$, total proteins $=$ $59.51 \mathrm{~g} / 1$, albumins $=29 \mathrm{~g} / 1$, ATGT Ig A $>200 \mathrm{U} / \mathrm{ml}$ and $\mathrm{Ig} \mathrm{G}=35.6 \mathrm{U} / \mathrm{ml}$ antibodies $(\mathrm{NV}<20 \mathrm{UI} / \mathrm{ml})$. The pathology findings further to upper digestive endoscopy and duodenal intestinal biopsy were (Fig. 3): massive chronic duodenitis with partial villous atrophy, which were suggestive of MARSH III A.

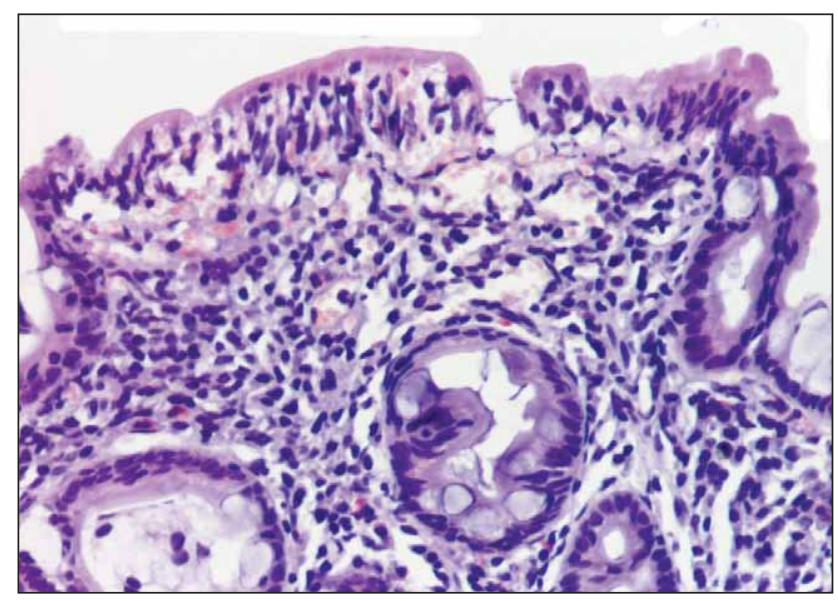

FIGURE 3. Chronic duodenitis with partial villous atrophy (MARSH III A)

In this case, the positive diagnosis was type I DM associated with CD and the twofold therapeutic approach included both a gluten-free diet and the actual type I DM management consisting of insulin therapy.

\section{DISCUSSIONS}

The authors reported four clinical cases of CD with symptoms such as recurrent abdominal pain, impaired hepatic function, constipation and one case of type I diabetes mellitus associated with celiac disease. Growth impairment was detected in all these patients, regardless of their clinical (constipation, recurrent abdominal pain), or biological (cytolytic hepatitis syndrome, hyperglycaemia with glycosuria) symptoms.

The first CD cases were reported by Arataeus of Cappadocia in the $1^{\text {st }}$ century BC. Much later, i.e. in 1888, Samuel Gee concluded that the disease affected children aged 1 to 5 years, being caused by some error in these children's diet. Although he described the clinical picture of the disease, Gee failed to see the fundamental connection between $\mathrm{CD}$ and gluten intake (On the Coeliac Affection, 1888) (2,3). In the 1940's, Willem Karen Dicke noticed the influence of wheat and related cereals on the triggering of CD symptoms and he was the first one to recommend the first and still only CD treatment: a gluten-free diet (4).

CD may occur at any age, from infants to aged individuals, its clinical signs varying depending on the patient's age on disease onset. Clinical signs are 
correlated particularly with the patient's age on disease onset, as they are often not considered as suggestive of this affliction (5).

The classical CD onset in children occurs around the age of 18 months, weeks and even months after cereals have been included in the children's diet. In babies, CD onset is insidious and consists of steatorrhea, doughy, faded, massive stools with bits of undigested food. The classical triad of chronic diarrhea, swollen abdomen and malnutrition is increasingly rarer. On the other hand, atypical forms have been more and more common (2). Among the atypical manifestations in our patients, there were found recurrent abdominal pains which delayed diagnosis until the age of 5, while constipation and hepatic cytolysis syndrome associated with malnutrition were diagnosed in the second year of life. Its early onset, during infancy, is characterized by vomiting and watery long-recovery stools. During the course of the disease, the patients may experience recurrent episodes of severe diarrhea, with watery stools and severe dehydration, or celiac attacks, which have been increasingly rarer. After the age of 3-5 years, the place of digestive symptoms on disease onset is taken by weight and later height growth stagnation. In older children, CD is one of the main causes of weight and height hypotrophy due to anorexia, malabsorption and intestinal nutrient losses. All patients presented failure to thrive. Growth disorders are associated with anorexia, recurrent abdominal pain, constipation, marked physical asthenia and neuro-behavioral changes (children are lethargic, introvert or, on the contrary, short-tempered, unhappy or even violent; their focus and academic performance are also affected). The consequences of intestinal malabsorption are fits of hypocalcemic tetany, osteopenia or osteoporosis, spontaneous fractures of long bones (vitamin D and calcium malabsorption), Beri-Beri neuritis, glossitis, stomatitis, cheilitis, tegument paleness (B group vitamins malabsorption), bruising, nosebleeds, gum and intestinal bleeds (vitamin K malabsorption), as well as dermatosis and hair dystrophy (vitamin A malabsorption). Tooth enamel erosion, recurrent aphthous ulcer, delayed tooth eruption, cheilitis, oral lichen planus, atrophic glossitis and xerostomia are oral CD signs which, if timely recognized, allow early diagnosis setting (6).

Although the current trend is late CD onset, in children aged 5 to 7 years, in some patients the disease is diagnosed even later, during their adolescence or even adulthood (3). CD adolescents suffer from delayed sexual maturity, hypo- or amenorrhea, which are consequences of the impact of the disease on their growth and development. CD diagnosed during adulthood is characterized by longterm consequences of intestinal malabsorption (refractory iron deficiency anemia, folic acid, vitamin and mineral deficiencies), accompanied by infertility, dermatitis herpetiformis, arthritis and arthralgia (lumbar spine, hip and knee joints are often impaired). Literature data also show higher rates of malignant illnesses, including intestinal lymphoma, squamous cell esophageal carcinoma, melanoma and adenocarcinoma, in $\mathrm{CD}$ patients than in the general population $(7,8)$.

The following clinical forms of $\mathrm{CD}$ have been acknowledged (adapted according to the OSLO 2013 classification):

- the classical form manifested by signs and symptoms due to intestinal malabsorption: chronic steatorrheic diarrhea, failure to thrive, abdominal distension, anorexia, coeliac attacks.

- the non-cassical form includes both gastrointestinal symptoms, which are not due to intestinal malabsorption (recurrent abdominal pain, vomiting, constipation, irritable bowel syndrome) and extra-intestinal symptoms (increased level of liver enzymes, treatmentrefractory anemia, recurrent aphthous stomatitis, height hypotrophy, tooth enamel erosion, hypogonadism and delayed puberty, arthritis, dermatitis herpetiformis, epilepsy with intracranial calcifications, ataxia, autism, depression). These patients are usually monosymptomatic or oligosymptomatic.

- the subclinical form, formerly known as silent or asymptomatic $C D$, in which the patient is asymptomatic, but their intestinal mucosa has specific intestinal lesions. This category also includes the latent $\mathrm{CD}$ form defined by the existence of normal intestinal mucosa in children with gluten intake who will develop the disease subsequently manifested due to their genetic predisposition (the only intestinal mucosa change is intraepithelial lymphocytes increase) (2). This form does not have enough clinical signs and symptoms that would justify routine serological CD testing.

- the potential form ("potential coeliac disease") in which the patients' intestinal biopsy is normal, but they have a high risk of CD, which is supported by the positive serology $(15,16)$.

- the refractory form characterized by the fact that patients experience persistent or recurrent signs and symptoms of intestinal 
malabsorption with intestinal villi atrophy, which are not improved by a strict glutenfree diet adopted for more than 12 months.

- non-coeliac gluten sensitivity is characterized by one or more clinical, immunological or morphological signs triggered by gluten intake in people in whom CD was ruled out; this category may include non-IgE mediated food allergy (9).

$\mathrm{CD}$ is associated with a series of autoimmune (type I DM, autoimmune thyroiditis, Sjorgen syndrome, psoriasis, alopecia areata) or non-autoimmune (selective IgA deficiency, Down syndrome, Williams syndrome, Turner syndrome, IgA nephropathy, neuro-psychiatric conditions like autism, depression, ataxia, epilepsy with intracranial calcifications) diseases, $1^{\text {st }}$ degree relatives of $\mathrm{CD}$ patients (3).

The association between type I DM and CD is very well documented and supported by common genetic predisposition. It has been noticed that some type I DM patients experience episodes of symptomatic hypoglycaemia with progressive insulin intake diminution, which are apparently not accounted for by smaller quantities of ingested food or more exercise, before CD diagnosis setting. The occurrence of hypoglycaemia and diminution of insulin intake may be suggestive of active $C D$ in diabetes patients or of low gluten-free diet compliance in already diagnosed patients. CD-specific gastrointestinal signs (diarrhea, abdominal distension) are rare in diabetic patients, in whom atypical isolated signs and symptoms predominate: sideropenic anemia, height hypotrophy, late puberty onset, epilepsy, hypertransaminasemia, dyspeptic symptoms, dermatitis herpetiformis or recurrent aphthous stomatitis (10).

Immunological screening tests are used for diagnosis purposes, in order to reduce the need for intestinal mucosa biopsy and to monitor the course of $\mathrm{CD}$ under treatment and check gluten-free diet compliance. According to ESPGHAN recommendations, intestinal biopsy in children with typical symptoms may not be performed if the IgA ATTG titer is 10 times higher than the upper normal limit and exhibits a CD-specific HLA genotype predisposition. Serological screening tests have shown that $\mathrm{CD}$ is currently much more common than it was initially believed, its prevalence rate being $1 \%$ of the general population $(11,12)$. Patient diagnosing using screening tests has shown a higher incidence of atypical or silent forms, whereas the classical diarrhea form has been less frequent $(13,14)$.

\section{CONCLUSIONS}

The casuistry presented highlights the delay in $\mathrm{CD}$ diagnosis due to atypical and associated pathologies. The usage of immunological tests in children with atypical manifestations or as a method of screening for risk groups increased the incidence of atypical or silent forms in older children and adolescents. Knowing the clinical polymorphism manifestations of CD enables early diagnosis and prevents long-term complications: osteoporosis, infertility, intestinal lymphoma.

\section{REFERENCES}

1. Fasano A., Araya M., Bhatnagar S. et al. International Societies of Pediatric Gastroenterology, Hepatology, and Nutrition consensus report on celiac disease, J Pediatr Gastroenterol Nutr. 2008; 47(2): 214-9

2. Walker-Smith John A. Celiac disease. In: W. Allan Walker, Peter R. Durie, J. Richard Amilton et al. Pediatric Gastrointestinal Disease, $3^{\text {th }}$ ed, B.C. Decker Inc., 2000, 727-45

3. Fasano A. Clinical Presentation of Celiac Disease in the Pediatric Population. Gastroenterol. 2005; 128: S68-73

4. Meresse B., Ripoche J., Heyman M. Celiac disease: from oral tolerance to intestinal inflammation, autoimmunity and lymphomagenesis. Mucosal Immunology (2009); 2: 8-23

5. Stănescu Popp Alina. Diagnostic în boala celiacă la copil, Editura Cartea Universitară, Bucureşti, 2006

6. Greetje J.T., Wieke H.M. Verbeek, Marco W.J. et al. The spectrum of celiac disease: epidemiology, clinical aspects and treatment. Nat. Rev. Gastroenterol. Hepatol. 2010; 7: 204-13

7. Green P.H.R. The Many Faces of Celiac Disease: Clinical Presentation of Celiac Disease in the Adult Population. Gastroenterol. 2005; 128: S74-78

8. Dewar D.H., Ciclitira P.J. Clinical features and diagnosis of celiac disease. Gastroenterol 2005; 128: S19-24

9. Ludvigsson J.F., Leffler D.A., Bai J. et al. The Oslo definitions for coeliac disease and related terms. Gut. 2013 January; 62(1): 43-52

10. Smyth D.J., Plagnol V., Walker N.M. et al. Shared and distinct genetic variants in type 1 diabetes and celiac disease. N Engl J Med 2008; 359: 2767-77

11. McGough N., Cummings J.H. Coeliac disease: a diverse clinical syndrome caused by intolerance of wheat, barley and rye. Proceedings of the Nutrition Society (2005); 64: 434-50

12. Roma E., Panayiotou J., Karantana H. et al. Changing Pattern in the Clinical Presentation of Pediatric Celiac Disease: A 30-Year Study. Digestion 2009; 80:185-191

13. Rampertab S.D., Pooran N., Brar P. et al. Trends in the presentation of celiac disease. Am. J. Med. 2006; 119: 355-9

14. Lo W., Sano K., Lebwohl B. Changing presentation of adult celiac disease. Dig Dis Sci. 2003; 48(2): 395-8 